\title{
Image-based guidance of percutaneous abdomen intervention based on markers for semi-automatic rigid registration
}

\author{
Dominik Spinczyk \\ Faculty of Biomedical Engineering, Silesian University of Technology, Zabrze, Poland
}

Videosurgery Miniinv 2014; 9 (4): 531-536

DOI: $10.5114 /$ wiitm.2014.45048

\begin{abstract}
Introduction: For percutaneous abdomen intervention (e.g. liver radiofrequency (RF) tumor ablation, liver biopsy), surgeons lack real-time visual feedback about the location of the needle on planning images, typically computed tomography (CT). One difficulty lies in tracking and synchronizing both the tool movement and the patient breathing motion. Aim: To verify the correspondence between rigid registration fiducial registration error signal and breathing phase. Material and methods: Designed markers that are clearly visible both in planning CT and on the patient during the intervention are proposed. Registration and breathing synchronization is then performed by a point-based approach. The method was tested in a clinical environment on 10 patients with liver cancer using 3D abdominal CT in the exhale position. Median rigid fiducial registration error (FRE) in the breathing cycle was used as a criterion to distinguish the inhale and exhale phase.

Results: The correlation between breathing phase and FRE value can be observed for every patient. We obtained mean median FRE equal to $9.37 \mathrm{~mm}$ in exhale positions and $15.56 \mathrm{~mm}$ in the whole breathing cycle.

Conclusions: The presented real time approach, based on FRE calculation, was integrated in the clinical pipeline, and can help to select the best respiratory phase for needle insertion for percutaneous abdomen intervention, in cases where only $3 D C T$ is performed. Moreover, this method allows semi-automated rigid registration to establish the correspondence between preoperative patient anatomical model and patient position.
\end{abstract}

Key words: percutaneous abdomen intervention, respiratory phase synchronization, real-time rigid registration.

\section{Introduction}

Due to different variants of patients' anatomy, minimally invasive surgery is a challenging task. That is why nowadays a big effort is made to develop image-guided navigation systems which allow taking into account spatial information of patient anatomy, obtained from different 2D/3D/4D medical images, during the interventions. The goal is to find the actual position of internal anatomical structures (depends on intervention) during the procedure and the correspondence with the preoperative anatomical model [1].
For percutaneous abdomen intervention (e.g. liver radiofrequency (RF) tumor ablation, liver biopsy), surgeons lack real-time visual feedbacks about the location of the needle on planning images, typically computed tomography (CT), and breathing is one of the most important causes of internal organ movement [1-3]. One difficulty lies in tracking and synchronizing both the tool movement and the patient breathing motion. Taking into account clinical and surgical circumstances of intervention and the difficulty to find and track some anatomical landmarks inside the body, the preoperative 4D image modality 
is used to create a breathing motion model which builds the connection between the breathing signal and anatomical organ movement [4]. Then during the intervention the breathing signal is reconstructed using different techniques [5] and the correspondence model between the surrogate breathing signal and 4D breathing motion model is established [4]. In some clinical cases only 3D CT is performed. The registration process in image-guided intervention is usually divided into two steps [1]:

- Rigid registration between Cartesian coordinate systems of patient anatomical model and tracker coordinate system in operating room.

- Non-rigid registration of patient position - taking into account deformation of patient's body during interventions.

The article is focused on the first step of the presented pipeline: the rigid registration. Meier-Hein segment needle shape markers in $\mathrm{CT}$ images track it $[6,7]$ attached to the swine [8] or on the human body breathing phantom [9] to establish the correspondence between preoperative patient 3D image and position during intervention.

\section{Aim}

The goal of this paper is to verify the correspondence between rigid registration fiducial registration error signal and breathing phase, which is useful for percutaneous abdomen interventions, in cases where only the $3 \mathrm{D}$ image, to build the preoperative anatomical model, is used.

\section{Material and methods}

Finding the correspondence between preoperative anatomical model and patient position in the operating room during the intervention is a big challenge so the divide and conquer paradigm is used. The first step is usually rigid registration, due to its lack of problem of ambiguity, low computational complexity and the fact that it is regarded as the first global coarse fit, gives orientations for the accuracy of the fit and can be regarded as a solution to the problem of initialization for the non-rigid registration algorithms [1]. To find rigid mapping between two Cartesian coordinate systems, data must include three or more corresponding non-collinear points. Horn et al. [10] proposed a closed form solution based on a least-squares formulation. Optimal rotation and translation matrices are found using singular value decomposition (SVD) of the correlation matrix:

$$
\operatorname{SVD}(C)=U \operatorname{diag}\left(\sigma_{i}\right) V
$$

where $\mathrm{C}=\sum_{i=1}^{n} \mathrm{~S}_{i}^{T} \mathrm{~T}_{i}$ is the correlation matrix, $\mathrm{Si}$ are points in the first Cartesian coordinate system, Ti are points in the second Cartesian coordinate system, $\sigma_{i}$ are non-negative singular values of the correlation matrix, and $U$ and $V$ are orthonormal matrices,

$$
\operatorname{Rot}=U\left[\begin{array}{ccc}
1 & 0 & 0 \\
0 & 1 & 0 \\
0 & 0 & \operatorname{det}\left(U V^{\top}\right)^{\prime}
\end{array}\right] V^{T}
$$

and

$$
\text { Trans }=\bar{T}-\operatorname{Rot}(\overline{\mathrm{S}})
$$

where $\bar{S}, \bar{T}$ are average values of the point coordinates in the first and second coordinate systems, respectively.

\section{Clinical implementation circumstances}

Implementation of this not complicated algorithm in the clinical environment is not a trivial task. A lot of circumstances should be taken into account. First of all both coordinate systems should be better defined. The first coordinate system is connected to the image volume acquired in the patient's preoperative diagnostic test: mostly CT or magnetic resonance (MR) modality. The second one in connected to the tracker coordinate system, which is mounted in the operating room. The basic requirement is that the markers should be visible in both coordinate systems. The idea is that the markers remain in the same position during the CT/MR scan and then they will be tracked by the tracking system. From the theoretical point of view the increased number of markers reduces the registration errors, but during the intervention we should follow clinical circumstances. Marker visibility in both coordinate systems means:

1. The positions of markers are in the tracking system working volume (working volume is defined by the manufacturer and this is a space where accuracy of tracking is guaranteed).

2. The optical and video metric tracking systems require a line of sight between the camera and markers.

3. The markers should be in the CT/MR field of view (field of view - space which is covered by image volume). 
4. The positions of the markers should not disturb intervention (e.g. ultrasound acquisitions during treatment).

The kind of markers which could be used depends on the used tracking system. Generally, tracking systems are divided into three groups: optical, video metric or electromagnetic [1]. The main advantage of the electromagnetic system compared to different kinds is that it does not require a line of sight between the camera and markers so it could be used inside the body (e.g. for tracking flexible tools such as endoscopes) but the accuracy of tracking is lower $[7,11,12]$.

From the Horn algorithm perspective, based on points correspondence, the ideal marker should be identified as a point in the same way in both the image and tracker coordinate system. In fact, the tracking system tracks the markers and returns their position, which should be the same position as the marker visible in image modality.

\section{Breathing motion problem during rigid registration}

The decision where the marker should be placed is not easy. On the one hand, placing the markers outside the patient avoids the breathing problem (e.g. above the patient), but it works only with the implicit assumption that the patient does not move between $\mathrm{CT}$ acquisition and intervention. In some cases of intervention (e.g. CT guided with patient fixation) it could be true, but in some cases the in tervention is performed in a different room - so the markers attached to the patient will be better in these cases.

Generally it is possible to detect positions of markers attached to the patient manually by touching it by tracking tools or automatically. In the manual case different markers are touched at different breathing moments. Another disadvantage is that it takes time, which increases the patient's stay in the operating room and may result in less accuracy due to the uniqueness of inter- and intra-observer manual touching and creeping errors because of registration markers not corresponding [13]. This is a reason to use automatic tracking and distinguish markers in proposed solutions.

\section{Proposed solution}

A solution based on the video metric tracking system Micron Tracker Hx 40 is proposed. To inte-

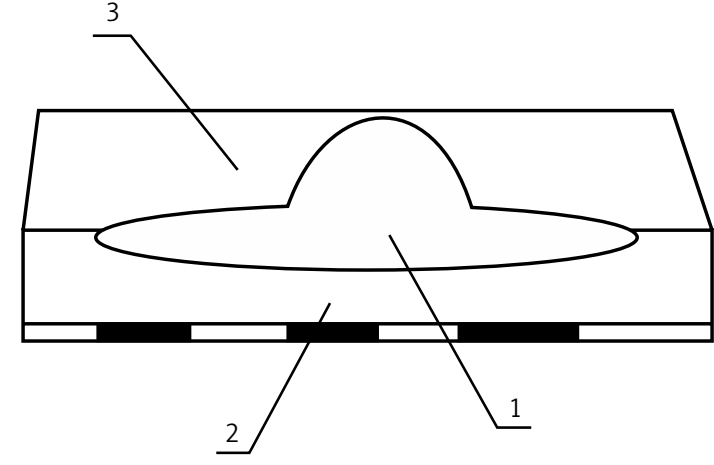

Figure 1. Integrated marker construction: part which is visible in CT (1), part which is visible by video metric tracking system (2), the adhesive element (3)

grate the Tracking System the OpenIGTLink protocol is used [14]. An integrated marker was designed [15] (Figure 1, Photo 1), which is visible in the $\mathrm{CT}$ image as a bright spot and could be tracked by a videometric tracking system. Considering liver tumor ablation, which takes place in the operating room in general anesthesia, the markers were attached to the patient.

To find the best rigid transformation between coordinate systems the following pipeline is proposed: 1. Attach the markers to the patient before CT acquisition,

2. Perform CT acquisition,

3. Find the position of each marker in the CT volume twice by two radiologists and find the average position (to minimize inter- and intra-observer errors),

4. Prepare the patient in general anesthesia for ablation in operating room,

5. Start tracking the markers on the patient's abdomen during respiratory breathing,

6. Compute Horn transformation and Fiducial Registration Error (FRE) for every time for which a set of marker positions in the tracking coordinate system is acquired,

7. Find the best transformation, which has minimal FRE.

The FRE is calculated following the equation:

$\operatorname{FRE}(S, T)=\frac{1}{N_{s}} \sum_{i=1}^{N_{s}}\left\|S_{i}-\left(\operatorname{Rot}_{j}\left(T_{i, j}\right)+\operatorname{Trans}_{j}\left(T_{i}\right)\right)\right\|^{2}$

where: $S_{i}$ - position of $i$-th market in CT coordinate system, $T_{i, j}$ - positions of $i$-th marker at the $j$-th 


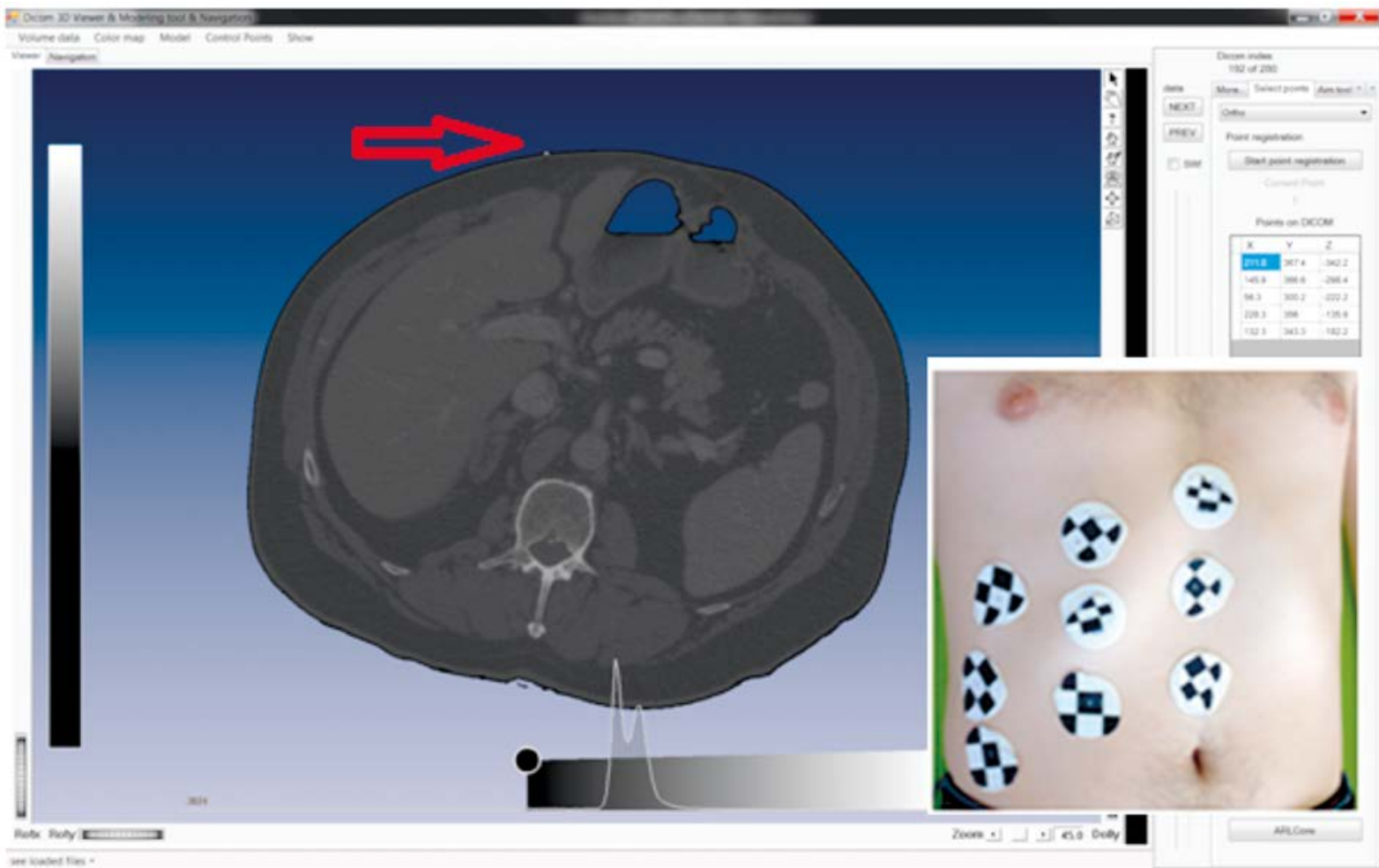

Photo 1. Patient with attached markers (right), which are visible in CT image (left) - with 9 markers attached to the patient

moment of time in tracker coordinate system, $\mathrm{N}_{\mathrm{S}}-$ number of markers, Rotj, Transj - Horn rotation and translation at the $j$-th moment of time.

Analyzing the time course and neighboring extremes of markers' position allows breathing cycles to be distinguished. Because breathing is a process variable over time, it is better to introduce a breathing phase $[16,17]$. A mean of maximum breathing phase is assigned by the formula (assuming zero breathing phase for minima) [16]:

$$
\bar{\emptyset}^{\max }=\frac{1}{N^{\max }} \sum_{i=1}^{N^{\max }} \frac{t_{i}^{\max }-t_{i}^{\min }}{t_{i+1}^{\min }-t_{i}^{\min }},
$$

where $N^{\text {max }}$ is the number of the observed breathing cycles and $t_{i}^{\max }$ and $t_{i}^{\min }$ are moments of time corresponding to the next extremes of marker position, and the respiratory phase, for any time tj is interpolated linearly [16]:

$\emptyset_{j}\left\{\begin{array}{c}\left(\frac{t_{j}-t_{i}^{\min }}{t_{i}^{\max }-t_{i}^{\min }}\right) \bar{\emptyset}^{\text {max }} ; \text { for } t_{i}^{\text {min }} \leq t_{j}<t_{i}^{\max } \\ \bar{\emptyset}^{\max }+\left(\frac{t_{j}-t_{i}^{\max }}{t_{i+1}^{\min }-t_{i}^{\max }}\right)\left(1-\bar{\emptyset}^{\max }\right) ; \text { for } t_{i}^{\max } \leq t_{j}<t_{i+1}^{\text {min }} .\end{array}\right.$
Calculating the breathing phase allows one to group breathing data from many breathing cycles and also compare data between patients.

\section{Results}

The presented approach was tested on an abdominal phantom and 10 patients' 3D abdomen $\mathrm{CT}$ with contrast agent in the exhalation breathing phase (GE Light Speed 16 Pro CT Scanner; Contrast medium: Iomeron 400 or Ultravist 370; Intravenous bolus: $10 \mathrm{ml}$ of $0.9 \% \mathrm{NaCl}, 1 \mathrm{ml} / \mathrm{kg}$ contrast medium and thereafter $30 \mathrm{ml}$ of $0.9 \% \mathrm{NaCl}$ all with $6 \mathrm{ml} / \mathrm{s}$ infusion speed) with liver metastases after approval of the Ethics Committee. The markers were attached to the patient before CT examination. Patients were diagnosed in the standard way to differentiate focal lesions using only three-dimensional computed tomography with contrast agent, without performing 4D CT. Usually on the same day in the next few hours markers attached to the patient are tracked in the operating room during free breathing without any patient shape conserving system before intervention. Markers were intentionally tracked during free 
breathing (before the general anesthetic) to make it possible to observe changes in the respiratory process and their impact on the calculation of FRE.

Figure 2 presents the time coarse of the marker position, breathing phase and FRE error for selecting patients. At the beginning of the time coarse of the normalized breathing phase (Figure 2) disturbance of the breathing phase calculation could be observed. In OR liver tumor ablation is usually performed in general anesthesia, so breathing is more regular than free breathing (disturbance of the breathing pattern can be excluded - see continued progress of time coarse of FRE error - Figure 2).

\section{Discussion}

Meier-Hein mentioned the correlation between FRE and breathing phase for the phantom of breathing motion and for the combination of internal and external marker attached to the swine $[8,9]$. One of the goals of the presented approach was to verify whether this correlation exists and could be used during treatment in the clinical environment. The correlation between breathing phase and FRE value can be observed for every patient. To summarize the results of the 10 patients, minimum, maximum and mean values of median FRE for inhalation, exhalation and continuous breathing cycle are presented in Table I [18]. Median rigid fiducial registration error in the breathing cycle was used as a criterion to distinguish an inhale and exhale phase. We wanted to treat patients in an 'automatic' way, which means that we don't check whether some markers are shifted between $\mathrm{CT}$ and tracker position acquisition, so we don't filter data of outbound observation (e.g. when the marker is outside of the tracking system working volume).

There is no effort to optimize the value of FRE by using any patient shape conserving system or optimize the configuration and number of the markers. It was validated that it is possible to use the proposed method in a clinical environment. Designed markers could be easy attach to the patient and could be easy to segment in the CT images. For dif-
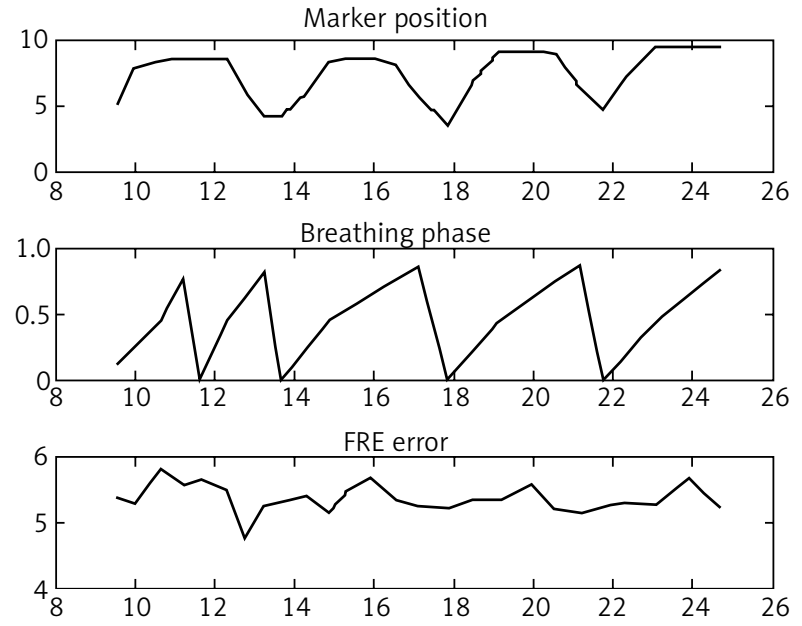

Figure 2. Time course of the marker position $(\mathrm{mm})$, breathing phase (normalized value) and FRE error $(\mathrm{mm})$ for selecting patient

ferent patients we obtained different levels of FRE errors, which could be caused by different breathing patterns and different circumstances of conserving patient position from $\mathrm{Ct}$ acquisition. In the future work the second one could be overcome by using same shape conserving systems. To follow different patterns of breathing for different patients the advantages of using automatic tracking to distinguish markers could be used. It allows one to use similar techniques proposed by Hong and Hashizume [13]. They define minimum requirements for proper registration which could be changed during the registration, including the number of markers, minimum registration error and verifying the registration error for different permutations of acquired marker position. Markers' relative position to the target point has a big impact on target registration error estimation, which was proved by West et al. [19]. If distinguished markers are tracked, the problems of registering no corresponding positions during intervention are minimized so we can track a bigger number of markers (Photo 1) and create combinations of tracked markers relative to the target point to find the optimal configuration from the target point of view.

Table I. Errors of patient's attached markers' rigid registration for median FRE [17]

\begin{tabular}{|lccccccccc|}
\hline Error type & \multicolumn{7}{c|}{ Median value of FRE [mm] } \\
\cline { 2 - 12 } & \multicolumn{1}{c}{ Min. } & \multicolumn{7}{c|}{ Max. } & \multicolumn{3}{c|}{ Mean } \\
\cline { 2 - 12 } & Exhale & Inhale & Total & Exhale & Inhale & Total & Exhale & Inhale & Total \\
\hline FRE_rigid & 2.27 & 3.09 & 3.07 & 32.8 & 52 & 51.69 & 9.37 & 16.75 & 15.56 \\
\hline
\end{tabular}




\section{Conclusions}

The presented real time approach, based on FRE calculation, was integrated in the clinical pipeline, and can help to select the best respiratory phase for needle insertion for percutaneous abdomen intervention, in cases where only 3D CT is performed. Moreover, this method allows semi-automated rigid registration to establish the correspondence between the preoperative patient anatomical model and patient position. Implementation of the semi-automated real-time registration method in clinical practice is easier because of shortening of preparation time in OR, no necessity of touching the patient, and no dependency on the physician's experience. In the next step the presented approach could be completed on the method to synchronize the breathing phase reconstructed from the external markers with a breathing signal and estimation of the target position from some kind of intraoperative imaging (e.g. CT, ultrasound).

\section{Acknowledgments}

The study was supported by National Science Center, Polad, Grant No UMO-2-\12/05/B/ST7/02136.

\section{References}

1. Peters T, Cleary K. Image-guided interventions: technology and applications. Springer, 2008.

2. Vikram S, Dogra E, Saad A. Ultrasound-guided procedures. Thieme, 2010.

3. Spinczyk D, Karwan A, Rudnicki J, Wróblewski T. Stereoscopic liver surface reconstruction. Videosurgery Miniinv 2012; 7: 181-7.

4. Ehrhardt J, Lorenz C. 4D Modeling and estimation of respiratory motion for radiation therapy. Springer, 2013.

5. McClelland J, Hawkes D, Schaeffter T, King A. Respiratory motion models: a review. Medical Image Analysis 2013; 17: 19-42.

6. Maier-Hein L, Pianka F, Seitel A, et al. Precision targeting of liver lesions with a needle-based soft tissue navigation system. Lecture Notes in Computer Science 2007; 4792: 42-9.

7. Maier-Hein L, Maleike D, Neuhaus J, et al. Soft tissue navigation using needle-shaped markers: evaluation of navigation aid tracking accuracy and CT registration. Proceedings of SPIE Medical Imaging 2007; 650900-26.

8. Maier-Hein L, Tekbas A, Franz A, et al. On combining internal and external fiducials for liver motion compensation. Computer Aided Surgery 2008; 13: 369-76.

9. Maier-Hein L, Muller S, Pianka F, et al. Respiratory motion compensation for CT-guided interventions in the liver. Computer Aided Surgery 2008; 13: 125-38.

10. Horn B, Hilden H, Negahdaripour S. Closed form solution of absolute orientation using orthonormal matrices. J Opt Soc Am A 1998; 5: 1127-35.
11. Web site manufacturer NDI measurement solutions in medical: http://www.ndigital.com/medical.

12. Web site manufacturer ClaronTech Micron Tracker specification: http://www.clarontech.com/microntracker-specifications.

13. Hong J, Hashizume M. An effective point-based registration tool for surgical navigation. Surg Endosc 2010; 24: 944-8.

14. Tokuda J, Fischer G, Papademetris X, et al. OpenIGTLink: an open network protocol for image-guided therapy environment. International Journal of Medical Robotics and Computer Assisted Surgery 2009; 5: 423-34.

15. Patent Application WIPO ST 10/C PL406230: marker and method for automatically positioning a patient in particular in the imaging navigation systems.

16. Rijkhorst E, Heanes D, Odille F, et al. Simulating dynamic ultrasound using MR-derived motion models to assess respiratory synchronisation for image-guided liver interventions. Lecture Notes in Computer Science 2010; 6135: 113-23.

17. Siebenthal von M, Szekely G, Lomax A, and Cattin P. Systematic errors in respiratory gating due to intrafraction deformations of the liver. Medical Physics 2007; 34: 3620-9.

18. Spinczyk D, Żyłkowski J, Wróblewski T. Continuous registration based on computed tomography for breathing motion compensation. Videosurgery Miniinv 2013; 8: 265-72.

19. West J, Fitzpatrick J, Toms S, et al. Fiducial point placement and the accuracy of point-based, rigid body registration. Neurosurgery 2001; 48: 810-6.

Received: 23.04.2014, accepted: 1.06.2014. 